Acta Crystallographica Section F

Structural Biology

and Crystallization

Communications

ISSN 1744-3091

Sabine Schneider, ${ }^{a}$ Maria J. V. M. Carneiro, ${ }^{\mathrm{b}}$ Charikleia loannou, Panos Soultanas ${ }^{\mathrm{b} *}$ and Max Paoli $^{\mathbf{a} *}$

${ }^{\mathrm{a}} \mathrm{School}$ of Pharmacy and Centre for Biomolecular Sciences, University of Nottingham, England, and ${ }^{\mathbf{b}}$ School of Chemistry and Centre for Biomolecular Sciences, University of Nottingham, England

Correspondence e-mail: panos.soultanas@nottingham.ac.uk, max.paoli@nottingham.ac.uk

Received 5 December 2006

Accepted 5 January 2007

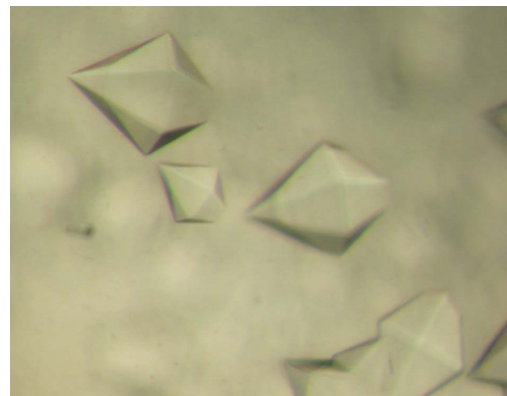

(C) 2007 International Union of Crystallography All rights reserved

\section{Crystallization and X-ray diffraction analysis of the DNA-remodelling protein DnaD from Bacillus subtilis}

The DnaD protein is an essential component of the chromosome-replication machinery of the Gram-positive bacterium Bacillus subtilis and is part of the primosomal cascade that ultimately loads the replicative ring helicase DnaC onto DNA. Moreover, DnaD is a global regulator of DNA architecture, as it forms higher order nucleoprotein structures in order to open supercoiled DNA. Here, the crystallization and preliminary X-ray diffraction analysis of the two domains of DnaD from B. subtilis are reported. Crystals of the N-terminal domain are trigonal, with either $P 3_{1} 21$ or $P 3_{2} 21$ space-group symmetry, and diffracted X-rays to $2.0 \AA$ resolution; crystals of the C-terminal domain are hexagonal, with space group $P 6_{1}$ or $P 6_{5}$, and diffracted X-rays to $2.9 \AA$ resolution in-house. Determination of the structure of the DnaD domains will provide insight into how remodelling of the nucleoid is associated with priming of replication in the model Gram-positive organism B. subtilis.

\section{Introduction}

Initiation of bacterial DNA replication involves the remodelling of a replication origin (oriC) through the action of the initiator protein DnaA and this is followed by a series of molecular events, known as the primosomal cascade, that ultimately result in the loading of two replicative ring helicases, one onto each strand of the duplex DNA. DNA primases are then recruited by helicases, thus signalling the switch from initiation to elongation; as a result, two replication forks start to migrate in opposite directions on the leading and the lagging strands (Fang et al., 1999; Marszalek \& Kaguni, 1994). Progression to the elongation stage does not guarantee completion of DNA replication, as the replication forks could accidentally be arrested anywhere along the DNA. In Escherichia coli, an active replication fork at arrested sites is reconstituted by the primosomal pathways, which include the PriA, PriB, PriC and DnaT proteins (Marians, 2000; $\mathrm{Ng} \&$ Marians, 1996), while priming at the oriC is mediated by DnaA. Both DnaA and PriA have homologues in Gram-positive bacteria such as Bacillus subtilis and other Gram-positive bacteria. In addition, another essential primosomal cascade involving the DnaD, DnaB and DnaI proteins is found in these Gram-positive bacteria. While DnaI is believed to be the Gram-positive functional homologue of the E. coli helicase-loader, both the DnaD and DnaB primosomal proteins have no homologues in Gram-negative bacteria and their exact functions are still being explored. They are essential for viability and are required for DnaA- and PriA-mediated initiation of DNA replication (Bruand et al., 2001; Moriya et al., 1999). At present, the molecular events of the priming mechanism are unclear, but data from various experiments indicate that DnaD interacts with DnaA, PriA and DnaB (Ishigo-Oka et al., 2001; Marsin et al., 2001), while DnaI interacts with the DnaC helicase (Imai et al., 2000; NoirotGros et al., 2002; Soultanas, 2002). DnaD is believed to act early in the cascade, setting the stage for the helicase recruitment by a helicaseloader constituted of both DnaB and DnaI (Velten et al., 2003). 
Table 1

Statistics for the processing and merging of the diffraction data.

Values in parentheses are for the highest resolution shell.

\begin{tabular}{lll}
\hline & DnaD N-domain & DnaD C-domain \\
\hline Space group & $P 3_{1} 21 / P 3_{2} 21$ & $P 6_{1} / P 6_{5}$ \\
Unit-cell parameters $(\AA)$ & & \\
$a(\AA)$ & 78.6 & 57.8 \\
$b(\AA)$ & 78.6 & 57.8 \\
$c(\AA)$ & 124.5 & 50.8 \\
$\alpha\left({ }^{\circ}\right)$ & 90.0 & 90.0 \\
$\beta\left({ }^{\circ}\right)$ & 90.0 & 90.0 \\
$\gamma\left({ }^{\circ}\right)$ & 120.0 & 120.0 \\
Wavelength $(\AA)$ & 1.5418 & 1.5418 \\
Resolution range $(\AA)$ & $28.0-2.0(2.1-2.0)$ & $15.0-2.9(3.1-2.9)$ \\
No. of observations & $177843(25869)$ & $22589(2331)$ \\
No. of unique reflections & $30662(4429)$ & $2161(294)$ \\
Multiplicity & $5.8(5.8)$ & $10.5(7.9)$ \\
Completeness $(\%)$ & $99.8(100.0)$ & $98.3(92.8)$ \\
$R_{\text {sym }} \dagger(\%)$ & $4.8(37.6)$ & $9.5(37.2)$ \\
Mean $I / \sigma(I)$ & $24.4(4.0)$ & $20.4(4.6)$ \\
Solvent content $(\%)$ & 62.9 or 25.8 & 39.7 \\
Matthews coefficient $\left(\AA^{3} \mathrm{Da}^{-1}\right)$ & 3.3 or 1.6 & 2.0 \\
Molecules per ASU & 2 or 4 & 2 \\
\hline
\end{tabular}

$\dagger R_{\text {sym }}=\sum_{h} \sum_{i}\left|I_{h, i}-\left\langle I_{h}\right\rangle\right| / \sum_{h} \sum_{i}\left|I_{h, i}\right|$, where the outer summation is over all unique $R_{\text {sym }}=\sum_{h} \sum_{i}\left|I_{h, i}-\left\langle I_{h}\right\rangle\right| / \sum_{h} \sum_{i}\left|I_{h, i}\right|$, where the outer summation is over all unique
reflections with multiple observations and the inner summation is over all observations of each reflection.

Recently, it was discovered that DnaD and DnaB have opposing global DNA-remodelling activities. DnaD interacts with linear DNA, forming a nucleoprotein structure with a round DnaD scaffold in the middle holding the DNA in an open circle around the periphery, whereas DnaB compacts laterally supercoiled and linear DNA (Turner et al., 2004; Zhang et al., 2005). Moreover, DnaD converts all the writhe of a supercoiled plasmid into twist (Zhang et al., 2005). The biological significance of these activities is not clear at present, but a functional model has been proposed with DnaD and DnaB being the link between global or local DNA remodelling and the initiation of DNA replication (Turner et al., 2004; Zhang et al., 2005). The function of DnaD is thought to be similar to the HU and H-NS proteins in E. coli. DnaD consists of two domains: oligomerization activity has been attributed to the $\mathrm{N}$-terminal domain (N-domain), while the C-terminal domain (C-domain) binds DNA and has an additional DNA-induced oligomerization activity (Carneiro et al., 2006). The untagged full-length protein is 232 residues in length and has a molecular weight of $27490 \mathrm{Da}$; the N-domain includes residues 1-128 and has a molecular weight of $16056 \mathrm{Da}$ (including the His tag) and the C-domain includes residues 129-232 and has a molecular weight of $13730 \mathrm{Da}$ (including the His tag). The sequence of DnaD has no significant similarity to any protein in the Protein Data Bank and therefore appears to have a unique signature. Revealing the structure-function relationships of the DNA-remodelling roles of DnaD is an aim of high biological significance as it will lead to a better understanding of how remodelling of the bacterial nucleoid is associated with replication priming in the Gram-positive model organism B. subtilis. Here, we report the crystallization and preliminary X-ray diffraction analysis of the two domains of DnaD.

\section{Methods}

\subsection{Cloning, expression and purification}

The N-domain and C-domain of DnaD from B. subtilis were cloned and purified by nickel-affinity chromatography as previously described (Carneiro et al., 2006). Briefly, E. coli BL21 (DE3) cells

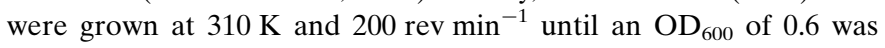
reached. Protein expression was then induced by adding IPTG to a final concentration of $1 \mathrm{~m} M$ and cultures were grown for a further $7-8 \mathrm{~h}$. The cells were lysed by sonication and the lysate was clarified by centrifugation. Purification was carried out in $50 \mathrm{~m} M$ phosphate buffer $\mathrm{pH} 7.5,100 \mathrm{mM} \mathrm{NaCl}$ using a HiTrap $\mathrm{Ni}^{2+}$-chelating column (Amersham Biosciences) and gel filtration in $50 \mathrm{mM}$ Tris $\mathrm{pH} 7.5$, $100 \mathrm{~m} M \mathrm{NaCl}, 1 \mathrm{~m} M$ DTT (Superdex 75, Amersham Biosciences). Protein samples in the gel-filtration buffer mentioned above were flash-frozen in liquid nitrogen for storage at $193 \mathrm{~K}$ after the addition of glycerol to a final concentration of $10 \%(v / v)$. Protein samples were thawed prior to crystallization and concentrated to $15-20 \mathrm{mg} \mathrm{ml}^{-1}$ using centrifugal membrane devices (Vivaspin) without exchanging the buffer.

\subsection{Crystallization}

Initially, high-throughput crystallization screening was carried out using the Hydra II micro-dispensing system (Robbins Hydra, Matrix Technologies Ltd, Wilmslow, Engand) with the sitting-drop vapourdiffusion method in 96-well microtitre plates (Greiner). Conditions from six crystallization screens, Classics, PEGs, $\mathrm{AmSO}_{4}$, Cations, MPDs and OptiSalts (Nextal Biotechnology/Qiagen), were tested with a protein concentration of $15-20 \mathrm{mg} \mathrm{ml}^{-1}$, a drop size of $0.6+$ $0.6 \mu \mathrm{l}$ and a reservoir volume of $100 \mu \mathrm{l}$. Crystallization plates were left undisturbed in a Sanyo MIR-253 incubator at $293 \mathrm{~K}$. Small single crystals $(0.07 \times 0.09 \times 0.03 \mathrm{~mm})$ of the $\mathrm{N}$-domain were obtained in $0.1 M$ sodium acetate $\mathrm{pH} 4.6,3.2 \mathrm{M}$ sodium chloride. Crystals of the $\mathrm{N}$-domain were improved by manual screening in sitting-drop vapour-diffusion experiments using 24-well Cryschem plates

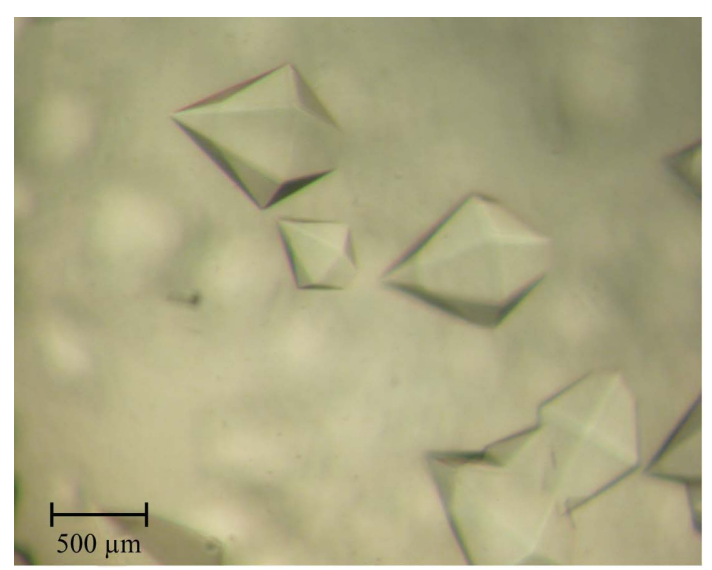

(a)

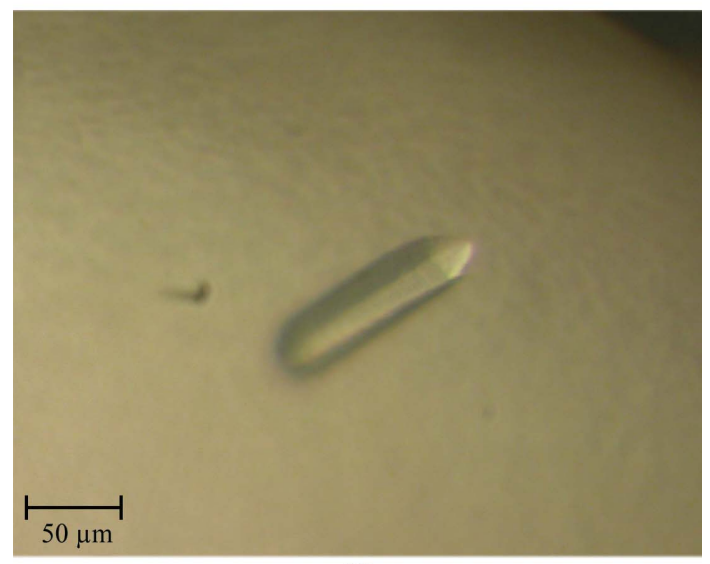

(b)

Figure 1

Crystals of $(a)$ the N-domain and $(b)$ the C-domain of DnaD. 


\section{crystallization communications}

(Hampton Research) with drop sizes of either $4+4 \mu \mathrm{l}$ or $8+8 \mu \mathrm{l}$ and a protein concentration of $20 \mathrm{mg} \mathrm{ml}^{-1}$.

\subsection{Data collection and analysis}

Prior to flash-freezing, crystals of the DnaD N-domain were transferred to cryoprotected artificial mother liquor containing $0.1 \mathrm{M}$ sodium acetate $\mathrm{pH}$ 4.6, $3.3 \mathrm{M}$ sodium chloride and $0.85 \mathrm{M}$ sodium malonate (Holyoak et al., 2003). C-domain crystals obtained in $2.2 \mathrm{M}$ ammonium sulfate and $0.2 \mathrm{M}$ sodium malonate were transferred to a cryoprotected artificial mother liquor consisting of $2.4 \mathrm{M}$ ammonium sulfate and $1 M$ sodium malonate prior to flash-freezing.

Data collections from both $\mathrm{N}$-domain and $\mathrm{C}$-domain crystals were carried out in-house using a Rigaku MicroMax 007 rotating-anode generator with a rotating copper anode (operating at $40 \mathrm{kV}$ and $20 \mathrm{~mA}$ ) equipped with VariMax HF optics (Osmic, Rigaku), an
R-AXIS IV++ image-plate detector and an X-stream 2000 cryocooling vapour jet. Diffraction data were processed using the programs MOSFLM v.6.2.4 (Leslie, 1992) and SCALA (Evans, 1997) as included in the $C C P 4$ suite (Collaborative Computational Project, Number 4, 1994).

\section{Results}

We have established conditions for the growth of diffraction-quality crystals of both the N-domain and C-domain of the B.subtilis DNAremodelling protein DnaD. The best results for the DnaD N-domain were obtained using 25-100 $\mathrm{m} M$ sodium acetate $\mathrm{pH} 4.6$ and 2.7-3.3 M sodium chloride and crystals typically appeared after 4-6 d and grew to dimensions of $0.8 \times 0.6 \times 0.6 \mathrm{~mm}$. (Fig. 1 a). Approximately two weeks after they first appeared, crystals of the N-domain started to

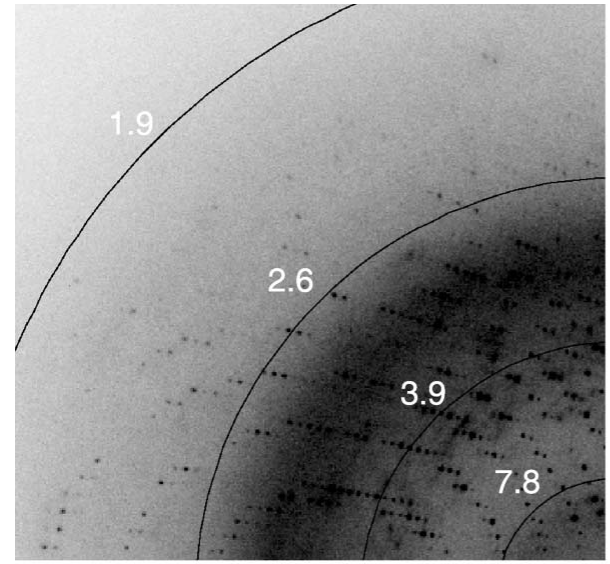

(a)

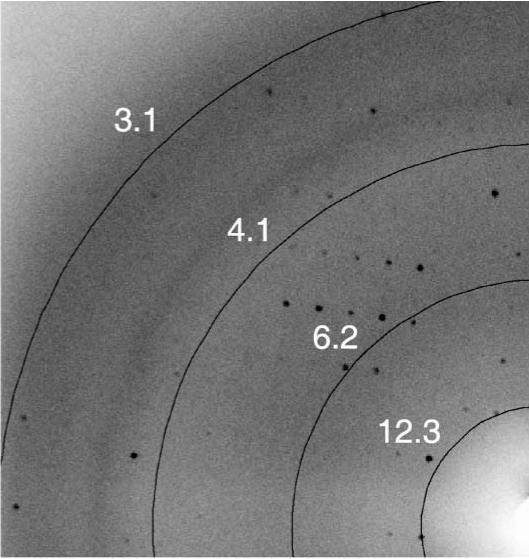

(b)

Figure 2

Diffraction pattern obtained from crystals of $(a)$ the N-domain and $(b)$ the C-domain of DnaD recorded using a home source.

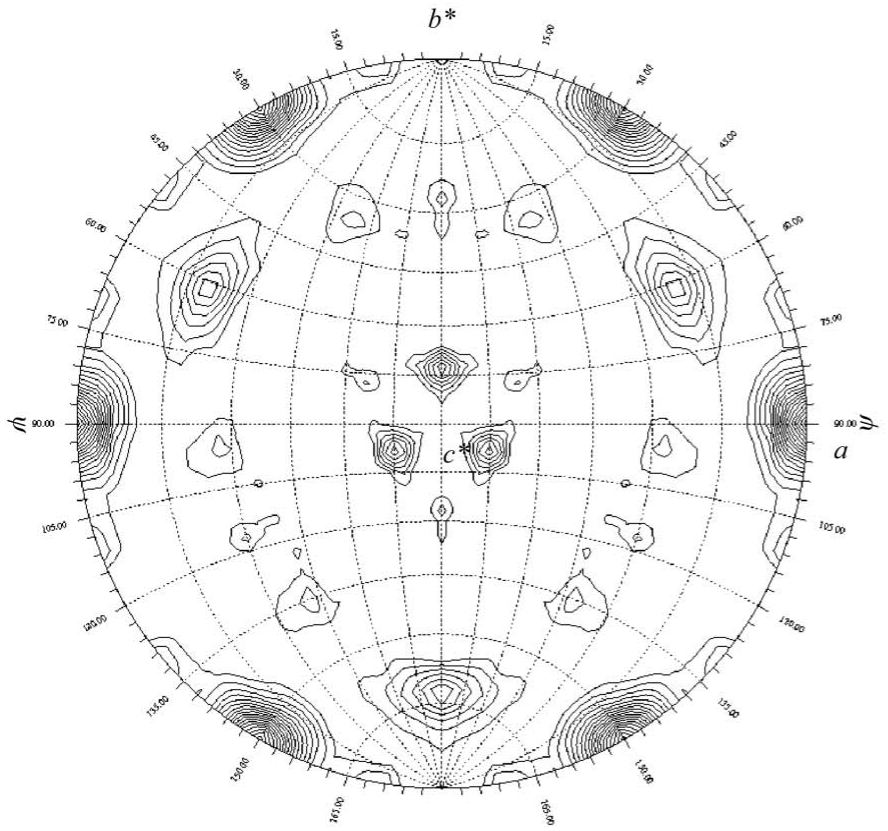

(a)

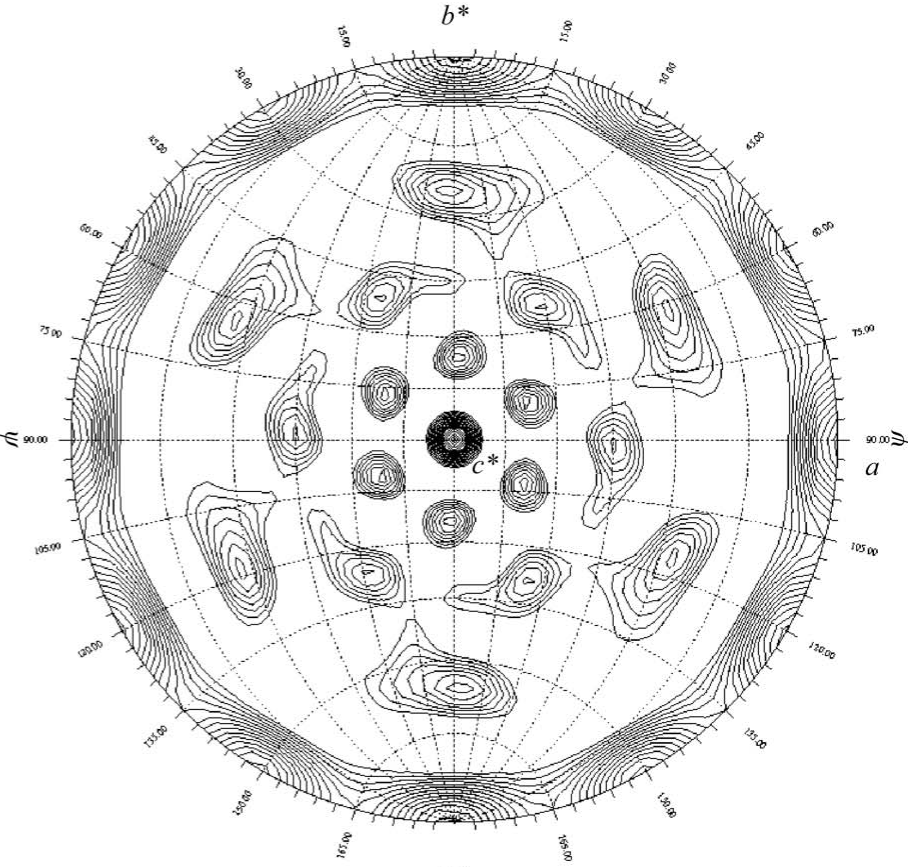

(b)

Figure 3

Self-rotation function of $(a)$ the N-domain and $(b)$ the C-domain calculated using the program GLRF (Tong \& Rossmann, 1997). In this analysis, data between 9 and $4 \AA$ resolution were used; the figure shows the section for $\kappa=180^{\circ}$ with a $20 \AA$ integration radius. 
lose their quality and degrade. Crystals of the C-domain (Fig. 1b) grew in various conditions containing ammonium sulfate, either by itself $(2.2 M)$ or in combination with one of the following salts (all at $0.2 \mathrm{M}$ ): ammonium fluoride, potassium acetate, potassium fluoride, sodium malonate, sodium sulfate decahydrate, sodium formate or sodium fluoride. Despite these crystallization conditions differing slightly, the C-domain crystals had similar shape and size: they were very small, $0.02 \times 0.02 \times 0.1 \mathrm{~mm}$, and required over four weeks to grow. As already performed for the $\mathrm{N}$-domain, the optimization of growth conditions for the C-domain crystals is in progress; the preliminary X-ray analysis reported below was carried out with C-domain crystals mounted straight from the 96-well plates.

The crystals are of sufficiently high quality to allow X-ray structure determination. The diffraction of the $\mathrm{N}$-domain crystals extended to $2.0 \AA$ resolution (Fig. $2 a$ ) and $100^{\circ}$ of data were collected with $0.5^{\circ}$ oscillations and an exposure time of $7 \mathrm{~min}$. The crystal of the C-domain diffracted to $2.9 \AA$ and a data set was collected consisting of 180 frames with $1^{\circ}$ oscillations and 12 min exposure times (Fig. $2 b$ ). The lattice symmetry of the N-domain crystals belongs to the enantiomorphic trigonal space groups $P 3_{1} 21$ or $P 3_{2} 21$, whereas the C-domain crystallizes with hexagonal symmetry and belongs to the enantiomorphic space groups $P 6_{1}$ or $P 6_{5}$. While our analysis was assisted by the program POINTLESS (Collaborative Computational Project, Number 4,1994), the diffraction data were also processed in alternative space groups, but the resulting high $R_{\text {sym }}$ values led us to rule out other possible symmetries. The statistics of the crystal unitcell parameters and data processing are shown in Table 1. Solventcontent and Matthews coefficient calculations on both domains suggest that two to four molecules pack in the asymmetric unit of the $\mathrm{N}$-domain, while one or two molecules are believed to make up the asymmetric unit of the C-domain crystals. A self-rotation function analysis on the data at $\kappa=180^{\circ}$ was carried out using the program GLRF (Tong \& Rossmann, 1997). This analysis indicates the presence of at least one twofold noncrystallographic symmetry axis for both the N-domain and C-domain lattices (Fig. 3). Thus, the $\mathrm{N}$-domain asymmetric unit is expected to consist of either a dimer or a tetramer, while based on solvent-content calculations the C-domain can only contain a dimer in the asymmetric unit. Determination of the structure of the N-domain using the SeMet MAD method should be possible given the presence of five methionine residues in its 128 amino-acid sequence. For the C-domain, which has no methionine residues, this method cannot be used unless mutations of leucine and/ or isoleucine residues to methionine are carried out. Elucidation of the structure of DnaD and/or of its domains will provide insights into the function of this unique protein essential for B. subtilis and lead to a better understanding of its role in nucleoid-remodelling associated with priming of DNA replication.

This work was supported by funds from the University of Nottingham and a BBSRC grant to PS and MP (grant reference BB/ E006450/1).

\section{References}

Bruand, C., Farache, M., McGovern, S., Ehrlich, S. D. \& Polard, P. (2001). Mol. Microbiol. 42, 245-255.

Carneiro, M. J., Zhang, W., Ioannou, C., Scott, D. J., Allen, S., Roberts, C. J. \& Soultanas, P. (2006). Mol. Microbiol. 60, 917-924.

Collaborative Computational Project, Number 4 (1994). Acta Cryst. D50, 760-763.

Evans, P. (1997). Jnt CCP4/ESF-EACMB Newsl. Protein Crystallogr. 33, $22-24$.

Fang, L., Davey, M. J. \& O'Donnell, M. (1999). Mol. Cell, 4, 541-553.

Holyoak, T., Fenn, T. D., Wilson, M. A., Moulin, A. G. \& Ringe, D. (2003). Acta Cryst. D59, 2356-2358.

Imai, Y., Ogasawara, N., Ishigo-Oka, D., Kadoya, R., Daito, T. \& Moriya, S. (2000). Mol. Microbiol. 36, 1037-1048.

Ishigo-Oka, D., Ogasawara, N. \& Moriya, S. (2001). J. Bacteriol. 183, 21482150.

Leslie, A. G. W. (1992). Jnt CCP4/ESF-EACMB Newsl. Protein Crystallogr. 26.

Marians, K. J. (2000). Trends Biochem. Sci. 25, 185-189.

Marsin, S., McGovern, S., Ehrlich, S. D., Bruand, C. \& Polard, P. (2001). J. Biol. Chem. 276, 45818-45825.

Marszalek, J. \& Kaguni, J. M. (1994). J. Biol. Chem. 269, 4883-4890.

Moriya, S., Imai, Y., Hassan, A. K. \& Ogasawara, N. (1999). Plasmid, 41, 17-29.

Ng, J. Y. \& Marians, K. J. (1996). J. Biol. Chem. 271, 15642-15648.

Noirot-Gros, M. F., Dervyn, E., Wu, L. J., Mervelet, P., Errington, J., Ehrlich, S. D. \& Noirot, P. (2002). Proc. Natl Acad. Sci. USA, 99, 8342-8347.

Soultanas, P. (2002). Nucleic Acids Res. 30, 966-974.

Tong, L. \& Rossmann, M. G. (1997). Methods Enzymol. 276, 594-611.

Turner, I. J., Scott, D. J., Allen, S., Roberts, C. J. \& Soultanas, P. (2004). FEBS Lett. 577, 460-464.

Velten, M., McGovern, S., Marsin, S., Ehrlich, S. D., Noirot, P. \& Polard, P. (2003). Mol. Cell, 11, 1009-1020.

Zhang, W., Carneiro, M. J., Turner, I. J., Allen, S., Roberts, C. J. \& Soultanas, P. (2005). J. Mol. Biol. 351, 66-75. 